\title{
INTERACCIÓN GENOTIPO-AMBIENTE DEL RENDIMIENTO Y CALIDAD DE GRANO Y TORTILLA DE HÍBRIDOS DE MAÍZ EN VALLES ALTOS DE TLAXCALA, MÉXICO
}

\author{
GENOTYPE-ENVIRONMENT INTERACTION OF YIELD AND GRAIN AND TORTILLA \\ QUALITY OF MAIZE HYBRIDS AT THE HIGHLANDS OF TLAXCALA, MÉXICO
}

\author{
María Gricelda Vázquez Carrillo ${ }^{1 \star}$, David Santiago Ramos ${ }^{1}$, Yolanda Salinas Moreno ${ }^{1}$, Israel Rojas Martínez ${ }^{2}$, \\ José L. Arellano Vázquez ${ }^{3}$, Gustavo A. Velázquez Cardelas ${ }^{3}$ y Alejandro Espinosa Calderón ${ }^{3}$
}

${ }^{1}$ Laboratorio de Calidad de Maíz, Campo Experimental Valle de México, Instituto Nacional de Investigaciones Forestales, Agrícolas y Pecuarias (INIFAP). Km
38.5 Carretera México-Texcoco. 56230, Chapingo, Edo. de México. ${ }^{2}$ Campo Experimental Tlaxcala-INIFAP. Km. 2.5 Carretera Tlaxcala-Santa Ana. 90800, Col.
Industrial, Tlaxcala. Tlax. ${ }^{3}$ Campo Experimental Valle de México-INIFAP. Km. 13.5 Carretera Los Reyes-Texcoco. 56250, Coatlinchán, Texcoco, Estado de México.

*Autor para correspondencia (gricelda_vazquez@yahoo.com)

\section{RESUMEN}

Actualmente la baja productividad del maíz (Zea mays L.) en los Valles Altos del Centro de México se debe a condiciones ambientales adversas de sequía, temperatura más alta que la usual y heladas tempranas. Para aumentar la productividad es necesario desarrollar variedades estables con rendimiento alto y que cumplan con las características físicas de grano, nixtamal y tortilla que demandan las industrias procesadoras. En este trabajo se determinó el efecto de la interacción genotipo $\mathrm{x}$ ambiente sobre el rendimiento, las características físicas del grano y la calidad de nixtamal y tortillade 20 híbridos pre-comerciales y comerciales de maíz cultivados durante el ciclo primavera-verano de 2009 en seis localidades de los Valles Altos de Tlaxcala, México. Se evaluó el rendimiento de grano, peso hectolítrico, peso de 100 granos (PCG), índice de flotación (IF), color de grano y harina, y la calidad de nixtamal y tortilla. Los resultados para rendimiento y características físicas del grano fueron analizados estadísticamente mediante el modelo de efectos principales aditivos e interacción multiplicativa (AMMI), y los de calidad de nixtamal y tortilla bajo un diseño completamente al azar. El modelo AMMI proporcionó buena descripción de la interacción genotipo $\mathrm{x}$ ambiente, $\mathrm{y}$ de la estabilidad de los 20 híbridos. Las condiciones de siembra y las ambientales modificaron las características físicas de los híbridos, especialmente el tamaño del grano y la dureza. Huamantla y Tlatempa fueron las mejores localidades en rendimiento (9.8 y $8.3 \mathrm{t}$ ha $^{-1}$ ), tamaño (PCG $>33 \mathrm{~g}$ ), dureza $(\mathrm{IF}<40 \%$ ) y calidad de tortilla. Nicolás Bravo, Emiliano Zapata y San Bartolomé Cuahuixmatlac fueron las localidades que más afectaron adversamente al rendimiento (3.8, $4.3,2.6 \mathrm{t} \mathrm{ha}^{-1}$ ) y al tamaño y dureza del grano (PCG $<33 \mathrm{~g}$, IF $>60 \%$ ). De los híbridos evaluados 15 cumplieron con las especificaciones de la industria de la masa y tortilla, pero ninguno cumplió las especificaciones de la industria de harina nixtamalizada.

Palabras clave: Zea mays, calidad de grano y tortillas, estabilidad del rendimiento, interacción genotipo $\mathrm{x}$ ambiente, modelo AMMI.

\section{SUMMARY}

Currently, the low productivity of maize (Zea mays L.) in the central highlands of México is due to adverse environmental conditions such as drought, higher than usual temperatures and early frosts. To increase productivity, it is necessary to develop stable maize varieties with high yield which can meet the quality characteristics of grain, nixtamal and tortilla demanded by the processing industry. In this study, we determined the effect of the genotype $\mathrm{x}$ environment interaction on grain yield and on the physical traits of grain, nixtamal and tortilla of 20 pre-commercial and commercial maize hybrids, grown during the $\mathbf{2 0 0 9}$ harvest season in six locations in the highlands of Tlaxcala, México. Grain yield, test weight, 100-grains weight (HGW), flotation index (FI), color of grain and flour, and nixtamal and tortilla quality were evaluated. The results for grain yield and grain physical traits were statistically analyzed using the model of additive main effects and multiplicative interactions (AMMI), while data of nixtamal and tortilla quality were analyzed under a completely randomized design. The AMMI model provided a good description of the genotype $x$ environment interaction and stability of the 20 hybrids. Both planting conditions and environment induced changes on the physical characteristics of hybrids, especially the grain size and hardness. Tlatempa and Huamantla were the best localities for yield $\left(9.8\right.$ y $\left.8.3 \mathrm{t} \mathrm{ha}^{-1}\right)$, grain size (HGW > $33 \mathrm{~g}$ ), hardness $(\mathrm{FI}<40 \%)$ and tortilla quality. Nicolás Bravo, Emiliano Zapata and San Bartolomé Cuahuixmatlac were the most adversely affected sites in terms of yield $\left(3.8,4.3,2.6 \mathrm{tha}^{-1}\right)$, grain size and hardness (HGW $<33 \mathrm{~g}$, FI $>60 \%$ ). Fifteen hybrids met the specifications for masa and tortilla industry but none met the specifications for nixtamalized flour industry.

Index words: Zea mays, AMMI model, genotype x environment interaction, grain and tortilla quality, stability of grain yield.

\section{INTRODUCCIÓN}

En el Estado de Tlaxcala el cultivo de maíz (Zea mays L.) ocupa el primer lugar entre los principales granos básicos que se producen. En 2010 se sembraron más de 120000 ha, de las cuales 104000 ha fueron de temporal (secano) y 16000 de riego, con un rendimiento medio de 2.35 y $3.84 \mathrm{t}$ $\mathrm{ha}^{-1}$, y una producción anual de 243000 y $62000 \mathrm{t}$, respectivamente (SIAP, 2012). En esta entidad el cultivo se desarrolla en áreas de mediana, buena y muy buena productividad ubicadas en los distritos de Calpulalpan, Tlaxcala y Huamantla (María et al., 2003). De acuerdo con María et al. (2003), la baja producción de maíz se debe a lo errático e irregular distribución de la lluvia, heladas tempranas, granizadas, profundidad del suelo, textura de la capa arable, pendiente y baja fertilidad de los suelos, alto grado de erosión, además del uso de variedades criollas de bajo rendimiento, tardías y susceptibles al acame. Por ello se requiere 
de genotipos que mantengan un comportamiento estable en diferentes localidades y años, además de un rendimiento alto, lo cual es factible en función del potencial genético del híbrido, principalmente (Arellano et al., 2011). La factibilidad de incrementar los rendimientos sin considerar las condiciones ambientales impredecibles, está supeditada en $60 \%$ al potencial genético del híbrido o variedad a sembrar y en $40 \%$ a las prácticas de manejo de cultivo (Arellano et al., 2010).

Gran parte del maíz producido en los Valles Altos ( > $2200 \mathrm{msnm}$ ) tiene problemas de comercialización debido a que produce masa y tortillas de colores grises que afectan su aceptabilidad por el consumidor (Salinas et al., 2007). Las industrias de la masa y la tortilla (IMT) y de harina nixtamalizada (IHN) son cada vez más exigentes en la demanda de materia prima de calidad diferenciada y acorde con sus procesos. La IHN demanda maíces de tamaño mediano a grande (peso de cien granos 'PCG' $>33 \mathrm{~g}$ ) que sea retenido en $90 \%$ en la criba de 6 / 64", con grano duro (índice de flotación 'IF' < $20 \%$ ), proporción de endospermo córneo superior a $48 \%$ y color de harina nativa superior a $77 \%$ de reflectancia, en tanto que la IMT demanda maíces duros e intermedios ( $\mathrm{IF}=13$ a $62 \%$ ), con un peso hectolítrico mayor o igual a $74 \mathrm{~kg} \mathrm{hL}^{-1}$, pérdida de sólidos durante la nixtamalización menor a $5 \%$ y rendimiento de tortilla de $1.5 \mathrm{~kg} \mathrm{~kg}^{-1}$ maíz (Vázquez et al., 2012).

Varios investigadores han demostrado que el mejor modelo estadístico para el análisis de estabilidad y la interacción genotipo $\mathrm{x}$ ambiente (IGA) es el modelo AMMI que incluye efectos principales aditivos e interacciones multiplicativas (Córdova, 1992). En maíz, este modelo ha demostrado su eficiencia en la selección de genotipos con énfasis en el rendimiento; Oikeh et al. (2004) aplicaron el modelo para evaluar la estabilidad de la concentración de hierro y zinc en el grano de maíz. En trigo (Triticum aestivum L.), este modelo se ha utilizado para evaluar la estabilidad de los parámetros de calidad del grano (Rharrabti et al., 2003; Hristov et al., 2010).

Para aumentar la productividad del maíz en los Valles Altos Centrales de México es necesario desarrollar variedades que además de ser estables y con un alto rendimiento, tengan la calidad de grano que los procesos de producción de harinas nixtamalizadas y tortillas demandan. Por ello en este trabajo se planteó determinar el efecto de la interacción genotipo $\mathrm{x}$ ambiente sobre el rendimiento, las características físicas del grano y la calidad nixtamalera-tortillera, de 20 híbridos pre-comerciales y comerciales de maíz cultivados en seis localidades de los Valles Altos del Estado de Tlaxcala, México.

\section{MATERIALES Y MÉTODOS}

Se evaluaron 20 híbridos (Cuadro 1) durante el ciclo primavera-verano de 2009, en seis localidades de los Valles Altos del Estado de Tlaxcala (Cuadro 2). La densidad de población fue de 50 a 60 mil plantas ha ${ }^{-1}$. Las fechas de siembra y cosecha, así como las dosis de fertilización se muestran en el Cuadro 2 (María et al., 2003). La oportunidad de aplicación fue de un cuarto del nitrógeno, todo el fosforo y potasio a la siembra, una segunda aplicación con la mitad del nitrógeno a los 50 a $55 \mathrm{~d}$ después de la siembra y el resto $30 \mathrm{~d}$ después, en función de la presencia de humedad superficial en el suelo (María et al., 2003).

Cuadro 1. Identificación de los 20 híbridos cultivados en seis localidades de Tlaxcala, México. PV-2009.

\begin{tabular}{llll}
\hline Clave & \multicolumn{1}{c}{ Denominación } & \multicolumn{1}{c}{ Situación } & $\begin{array}{c}\text { Organismo o } \\
\text { empresa }\end{array}$ \\
\hline 1 & 'H-58' & Experimental & INIFAP \\
2 & 'H-66' & Comercial & INIFAP \\
3 & 'H-64' & Experimental & INIFAP \\
4 & 'H-68' & Experimental & INIFAP \\
5 & 'H-70' & Comercial & INIFAP \\
6 & 'H-47' & Experimental & INIFAP \\
7 & 'H-49' & Experimental & INIFAP \\
8 & 'H-51' & Experimental & INIFAP \\
9 & 'H-55' & Experimental & INIFAP \\
10 & 'HB-PRECOZ-2' & Experimental & INIFAP \\
11 & 'H-40' & Comercial & INIFAP \\
12 & 'H-52' & Comercial & INIFAP \\
13 & 'H-48' & Comercial & INIFAP \\
14 & 'BUHO' & Comercial & MONSANTO \\
15 & 'PROMESA' & Comercial & COLPOS \\
16 & 'H-50' & Comercial & INIFAP \\
17 & 'AS-722' & Comercial & ASPROS \\
18 & 'PROSPECTO AE3' & Experimental & INIFAP \\
19 & 'H-72' & Experimental & INIFAP \\
20 & 'NIEBLA' & Comercial & CERES \\
\hline
\end{tabular}

\section{Rendimiento y calidad de grano}

En campo se evaluó el rendimiento, representado por el peso de mazorca por parcela a la cosecha, por el peso de materia seca del grano ajustado a $14 \%$ de humedad, convertido a t ha ${ }^{-1}$ de grano mediante la proporción de grano en la mazorca y el factor que lo extrapola a la superficie de una hectárea. En el laboratorio se evaluó: peso de 100 


\begin{tabular}{|c|c|c|c|c|c|}
\hline Localidad & Ubicación geográfica ${ }^{\dagger}$ & Altitud (m) & Condición de siembra & Tipo de & \\
\hline Tlatempa (TL) & $\begin{array}{l}19^{\circ} 20^{\prime} \mathrm{LN} \\
98^{\circ} 12^{\prime} \mathrm{LO}\end{array}$ & 2259 & Punta de riego & \multicolumn{2}{|c|}{ Pluvisol, pH 7} \\
\hline Cuahuixmatlac (BA) & $\begin{array}{l}19^{\circ} 16^{\prime} \mathrm{LN} \\
98^{\circ} 05^{\prime} \mathrm{LO}\end{array}$ & 2839 & Humedad residual & \multicolumn{2}{|c|}{ Andosol, pH 5} \\
\hline Huamantla (HU) & $\begin{array}{l}19^{\circ} 19^{\prime} \mathrm{LN} \\
97^{\circ} 55^{\prime} \mathrm{LO}\end{array}$ & 2500 & Punta de riego & \multicolumn{2}{|c|}{ Regosol, pH 6.5} \\
\hline Nicolás Bravo (NB) & $\begin{array}{l}19^{\circ} 25^{\prime} \mathrm{LN} \\
97^{\circ} 57^{\prime} \mathrm{LO}\end{array}$ & 2513 & Temporal estricto & \multicolumn{2}{|c|}{ Cambisol, pH 6.5} \\
\hline Papalotla (PA) & $\begin{array}{l}19^{\circ} 10^{\prime} \mathrm{LN} \\
98^{\circ} 10^{\prime} \mathrm{LO}\end{array}$ & 2334 & Temporal estricto & \multicolumn{2}{|c|}{ Regosol, pH 6} \\
\hline \multirow[t]{3}{*}{ Emiliano Zapata (EZ) } & $\begin{array}{l}19^{\circ} 33^{\prime} \mathrm{LN} \\
97^{\circ} 55^{\prime} \mathrm{LO}\end{array}$ & 2900 & Humedad residual & \multicolumn{2}{|c|}{ Andosol, pH 5} \\
\hline & \multirow{2}{*}{$\begin{array}{c}\text { Fecha de } \\
\text { siembra/cosecha } \\
(2009)\end{array}$} & \multirow{2}{*}{$\begin{array}{c}\text { Fertilización } \\
\left(\mathrm{kg} \mathrm{ha}^{-1} \text { de N-P-K) }\right.\end{array}$} & \multirow{2}{*}{$\begin{array}{l}\text { Precipitación }{ }^{\dagger \dagger} \\
(\mathrm{mm})\end{array}$} & \multicolumn{2}{|c|}{$\mathrm{T}\left({ }^{\circ} \mathrm{C}\right)^{\uparrow}$} \\
\hline & & & & Máx. & Mín. \\
\hline Tlatempa (TL) & 09-05/25-11 & $160-60-45$ & 748 & 25.2 & 10.4 \\
\hline Cuahuixmatlac (BA) & $11-03 / 04-12$ & $140-60-30$ & 329 & 24.4 & 6.2 \\
\hline Huamantla (HU) & 30-04/19-11 & $160-60-45$ & 716 & 22.9 & 6.0 \\
\hline Nicolás Bravo (NB) & $19-05 / 02-12$ & $140-60-30$ & 760 & 21.2 & 9.1 \\
\hline Papalotla (PA) & $13-05 / 26-11$ & $140-60-30$ & 813 & 24.3 & 10.9 \\
\hline Emiliano Zapata (EZ) & $07-03 / 07-12$ & $140-60-30$ & 664 & 13.5 & 7.1 \\
\hline
\end{tabular}

${ }^{\dagger} \mathrm{LN}$ = latitud norte; $\mathrm{LO}=$ longitud oeste; ${ }^{\dagger+}$ Precipitación total; "Máx. = temperatura máxima, Mín. = temperatura mínima (datos presentados sólo durante el periodo de crecimiento de la planta (0 de siembra a cosecha). Fuentes: CONAGUA (2012); INIFAP (2012).

granos (PCG) (Billeb y Bressani, 2001), peso hectolítrico (PH) (método 84-10; AACC, 2000), índice de flotación (IF) como medida indirecta de la dureza del grano (Vázquez et al., 2011) y color en grano y harina con un reflectómetro Agtron M300A ${ }^{\circledR}$ (San Jose CA, USA) operado en modo verde y calibrado con los mosaicos 10 y 63 (método 14-30; AACC, 2000). El color en harina solo se midió en muestras con reflectancia en grano mayor a $55 \%$. Las proporciones de pedicelo, pericarpio, germen y endospermo se determinaron mediante la metodología de Billeb y Bressani (2001).

\section{Calidad de nixtamal y tortilla}

La calidad de nixtamal y tortillas se determino en muestras de dos localidades en donde se obtuvo el mayor y menor rendimiento, respectivamente. La nixtamalización y elaboración de las tortillas se hizo mediante el método descrito por Vázquez et al. (2011). El porcentaje de sólidos se cuan- tificó en el agua de cocción y de vavado("nejayote") despues de la nixtamalización y el reposo, y el pericarpio retenido se determinó mediante método gravimétrico (Vázquez et al., 2011). En nixtamal, masa y tortillas se evaluó la humedad por el método 44-10 del AACC (2000). Se cuantificó el rendimiento de tortilla como la cantidad de tortilla obtenida por kilogramo de maíz procesado. En tortillas frías se evaluó la fuerza de ruptura con el texturómetro Brookfield $\mathbb{R}$ modelo CT3 (Middleboro, MA, USA), después de 2 y $24 \mathrm{~h}$ de elaboradas (Arámbula et al., 2004). El color en tortillas se obtuvo con el colorímetro Agtron ${ }^{\circledR}$ calibrado con los mosaicos números 10 y 52 (Vázquez et al., 2012) y con el colorímetro Hunter Lab MiniScan XE Plus Modelo 45/0L ${ }^{\circledR}$ (Reston, VA, USA) en escala Cielab, con iluminante $\mathrm{D} / 65$ y un ángulo de $10^{\circ}$. En el colorímetro Hunter Lab se obtienen los valores de luminosidad (L), $\mathrm{a}^{\star} \mathrm{y} \mathrm{b}^{*}$; los de $\mathrm{a}^{*} \mathrm{y}$ $\mathrm{b}^{\star}$ sirven para determinar el ángulo de tono "hue" $\left(\mathrm{h}^{\circ}\right)$ y la pureza de color "croma" (C), variables que ubican el color del material en el plano dentro de las diferentes tonalidades 
del color rojo, amarillo, verde y azul (Salinas et al., 2012); como todos los híbridos son de color blanco, sólo se tomó en cuenta la variable $\mathrm{L}$.

\section{Análisis estadístico}

Los resultados de rendimiento de grano y características físicas se analizaron con el modelo AMMI (Zobel et al., 1988). La reflectancia (color) de harina y las variables de nixtamalización se analizaron con un diseño completamente al azar, prueba de comparación de medias (Tukey) y análisis de correlación, mediante el paquete estadístico SAS para Windows, versión 9.0 (SAS Institute, 2000).

\section{RESULTADOS Y DISCUSIÓN}

El análisis de varianza mostró que los cuadrados medios de ambientes, genotipos y la interacción genotipo $\mathrm{x}$ ambiente (IGA) fueron significativos $(\mathrm{P}<0.01)$ para rendimiento de grano, PH e IF. En el PCG y el color de grano, la IGA fue significativa $(\mathrm{P}<0.05)($ Cuadro 3$)$.

\section{Estabilidad del rendimiento}

Al descomponer la suma de cuadrados de la IGA en componentes principales (CP), se constató que sólo los valores propios asociados a los dos primeros ejes fueron significativos en la explicación de la variabilidad de la IGA, al acumular entre los dos $77.1 \%$ de la variabilidad total (Cuadro 3). El CP1 capturó $62.0 \%$ de la suma de cuadrados (SC) y 24.2 $\%$ de los grados de libertad (GL) debida a la IGA. El CP2 sólo representó $15.2 \%$ de la SC de la interacción, por lo que el CP1 constituye un parámetro valido para el estudio de la estabilidad del rendimiento de los híbridos evaluados en el presente trabajo. Los ejes 3, 4, 5 y 6 no fueron significativos, por lo que se agruparon en el residuo.

De acuerdo con Zobel et al. (1988), en la gráfica de CP el desplazamiento a través del eje de la abscisa refleja las diferencias en los efectos principales (genotipos y ambientes), mientras que el desplazamiento a través de la ordenada manifiesta las diferencias en el efecto de las interacciones, los genotipos o ambientes que aparecen en línea perpendicular al eje de la abscisa tienen medias similares, y los que caen en línea horizontal a la abscisa tienen patrones de IGA similar; los genotipos con valores altos positivos o negativos del $\mathrm{CP} 1$ presentan una alta IGA y, consecuentemente, aquellos con valores cercanos a cero tienen poca interacción.

La Figura 1A muestra que los híbridos con baja IGA (más estables) fueron 'Prospecto AE3' [18], 'H-55' [9] y 'H-47' [6], pero sus rendimientos fueron menores a la media de $5.6 \mathrm{t} \mathrm{ha}^{-1}$. El híbrido con una baja IGA y con un rendimiento superior a la media fue 'H-51' [8]. Los híbridos 'H-66' [2], 'H-52' [12] y 'HB-PRECOZ - 2' [10] mostraron una alta IGA y los rendimientos más bajos. El híbrido 'H-40' [11] presentó el rendimiento más alto, pero fue inestable a través de localidades.

Las localidades Huamantla (HU) y Tlatempa (TL) se caracterizaron como ambientes de altos rendimientos. Por la longitud de los vectores, el ambiente que mejor discriminó a los genotipos en la evaluación fue el de TL, seguido de Emiliano Zapata (EZ) y HU; en cambio, las localidades Nicolás Bravo (NB), Papalotla (PA) y Cuahuixmatlac (BA) no discriminaron bien entre genotipos. El mejor híbrido para el ambiente de EZ fue el ' $\mathrm{H}-51$ ' [8], para TL el 'H-40' [11] y para $\mathrm{HU}$ el ' $\mathrm{H}-48$ ' [13]. Los seis ambientes en donde se evaluaron los genotipos formaron tres grupos (Figura 1A): el primero agrupó a $\mathrm{HU}$ y TL, con rendimientos medios

Cuadro 3. Partición de la suma de cuadrados y cuadrados medios del modelo AMMI de 20 híbridos de maíz cultivados en seis localidades de Tlaxcala, México. PV-2009.

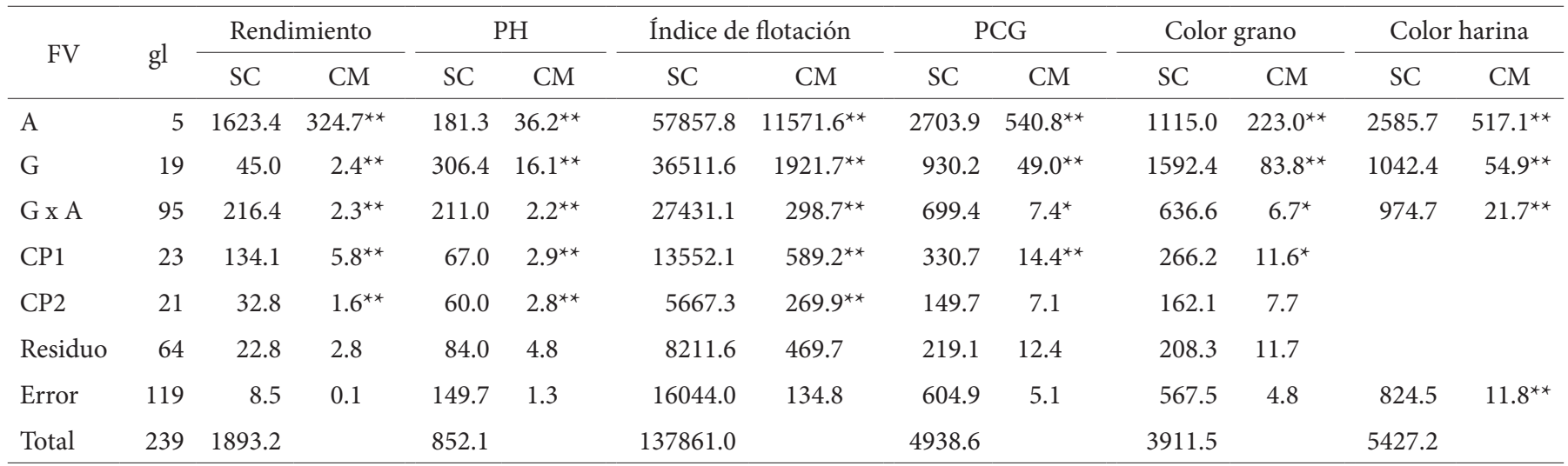

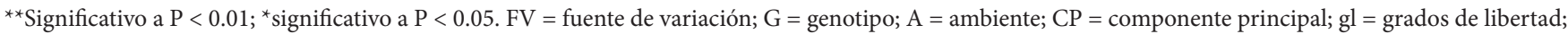
$\mathrm{PH}=$ peso hectolítrico; $\mathrm{PCG}=$ peso de 100 granos; $\mathrm{SC}=$ suma de cuadrados; $\mathrm{CM}=$ cuadrados medios. 

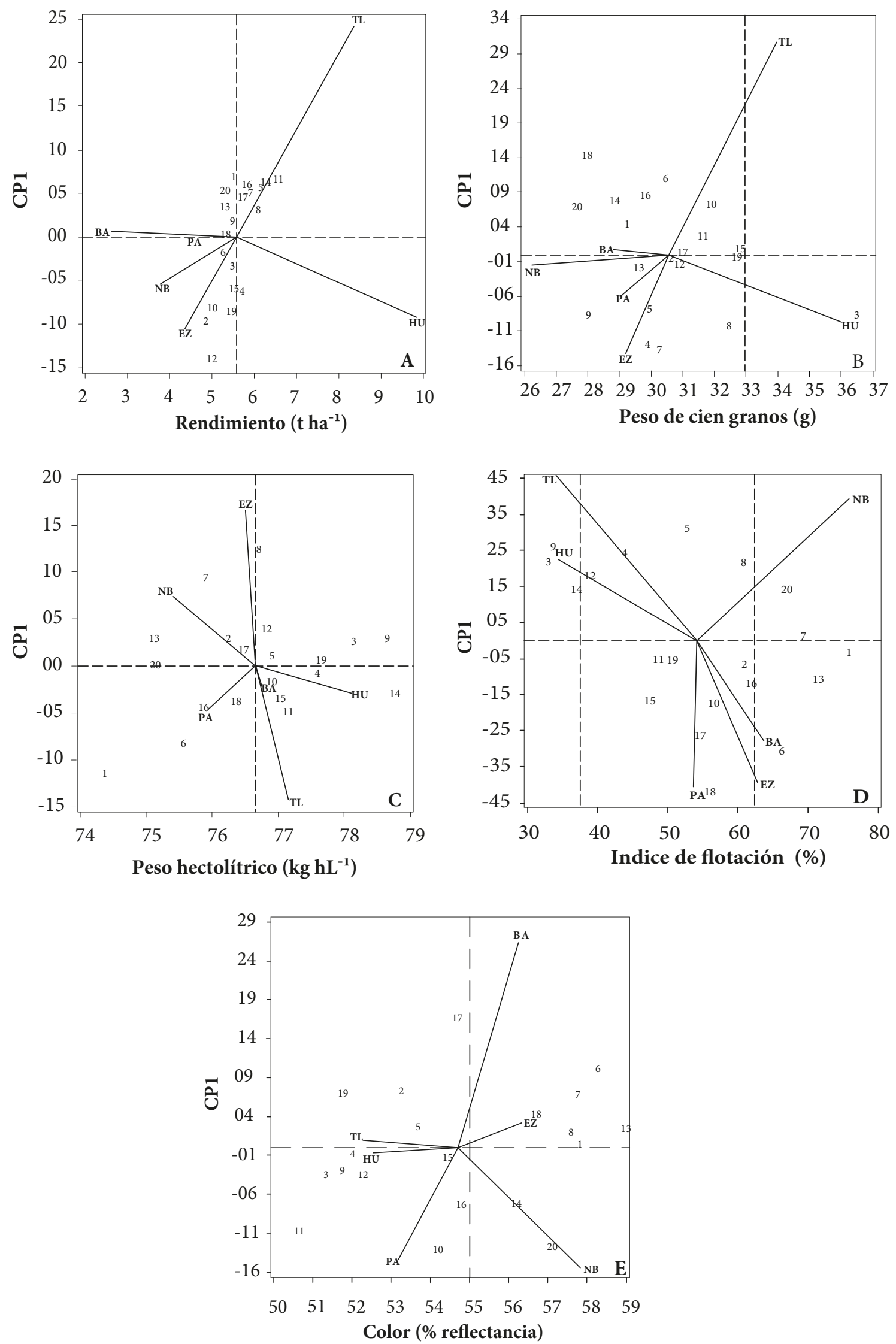

Figura 1. Promedios de rendimiento de grano (A), peso de cien granos (B), peso hectolítrico (C), índice de flotación (D), color de grano E y coeficientes del CP1 para la interacción de 20 híbridos de maíz y seis localidades del Estado de Tlaxcala, México. PV-2009. 
de 9.8 y $8.3 \mathrm{t} \mathrm{ha}^{-1}$, respectivamente; el segundo a EZ, NB y PA con medias de 4.3, 3.8 y $4.5 \mathrm{t} \mathrm{ha}^{-1}$, respectivamente; $y$ el tercero a BA con el rendimiento más bajo $\left(2.6 \mathrm{t} \mathrm{ha}^{-1}\right)$.

Los altos rendimientos obtenidos en HU y TL fueron resultado de una buena humedad del suelo al sembrar (en ambas localidades se sembró con un riego inicial -("punta de riego"), mayor precipitación pluvial y buena distribución de lluvia, temperaturas óptimas durante todo el desarrollo de la planta (Figura 2), una mayor dosis de $\mathrm{N}$ y $\mathrm{K}$ en la fertilización, y suelo profundo con un $\mathrm{pH}$ neutro o cercano a la neutralidad (Cuadro 2), que de acuerdo con The et al. (2006) son los mejores suelos para el maíz. Los rendimientos bajos en Nicolás Bravo y Papalotla fueron influenciados por suelos poco profundos en el caso de $\mathrm{NB}$ y arenoso en $\mathrm{PA}$, con $\mathrm{pH}<6.5$, bajas dosis de $\mathrm{N}$ y $\mathrm{K}$, y una baja y mala distribución de la precipitación, ya que cerca de $40 \%$ de la lluvia se presentó en el mes de septiembre, después de la etapa crítica de llenado del grano.

En el sitio EZ los rendimientos bajos fueron causados por la baja y mala distribución de la precipitación, además de temperaturas bajas $\left(<15^{\circ} \mathrm{C}\right)$ durante el periodo de llenado de grano (Figura 2). Por su parte en Cuahuixmatlac el rendimiento muy bajo fue resultado de altas temperaturas y sequía durante todo el periodo de crecimiento de la planta (Figura 2), ya que según Rincón et al. (2006), por cada grado centígrado que se incrementa la temperatura por encima del óptimo $\left(25^{\circ} \mathrm{C}\right)$, el rendimiento del grano se reduce en 3 a $4 \%$, debido a que la combinación de estos dos factores (alta temperatura y baja precipitación) causa una reducción en la fotosíntesis y una disminución del número de gránulos de almidón; aunado a lo anterior hubo efecto del suelo (ácido), erosionado y una baja dosis de fertilización.

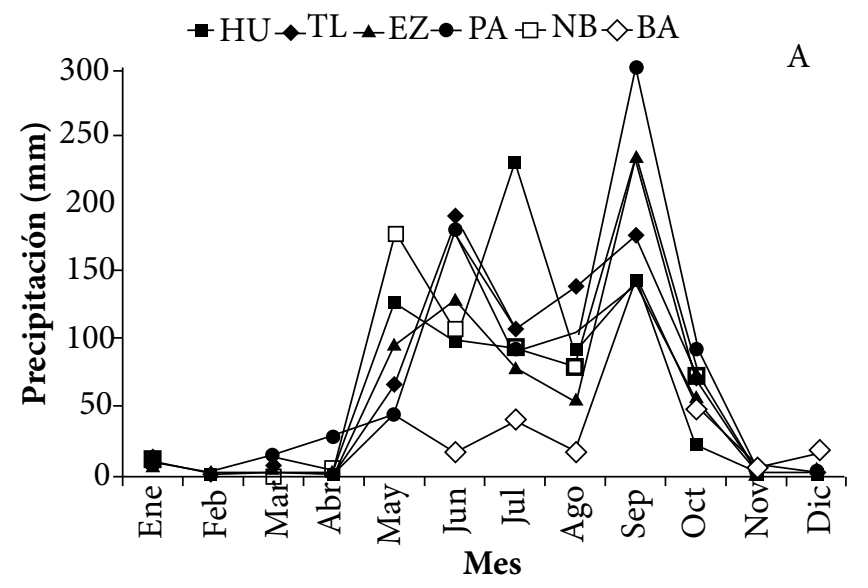

\section{Estabilidad de las características físicas de grano}

El análisis AMMI para el PCG mostró que 18.8 \% de la SC total fue atribuible a efectos genotípicos, mientras que los efectos ambientales y la IGA representaron 54.7 y 14.1 $\%$ respectivamente (Cuadro 3). Sólo el CP1 fue significativo y explicó $47.3 \%$ de la interacción, lo que demuestra que el efecto de la IGA fue explicado en su totalidad por el componente uno. Los híbridos 'H-72' [19], 'AS-722' [17], 'Promesa' [15], 'H-66' [2], 'H-52' [12], 'H-48' [13] y 'H-40' [11] presentaron los valores absolutos más bajos del $\mathrm{CP} 1$, por lo que se consideran los más estables en cuanto a PCG, a través de todos los ambientes (Figura 1B). El genotipo 'H-64' [3] fue el de mayor PCG a través de ambientes y por tanto el de mayor tamaño de grano, ya que según Billeb y Bressani (2001), el PCG es un indicador del tamaño de grano, y entre mayor sea el PCG mayor será el tamaño del grano. De acuerdo con la clasificación de Salinas et al. (2010), los restantes 19 híbridos presentaron granos de tamaño pequeño por tener un $P C G<33 \mathrm{~g}$.

El comportamiento de los ambientes en cuanto al tamaño de grano fue similar al rendimiento, y ambas variables correlacionaron entre sí $\left(\mathrm{r}=0.69^{* *}\right)$; los ambientes de HU y TL propiciaron un tamaño mayor de grano, mientras que en los demás ambientes los granos fueron pequeños. Uno de los principales factores que influye en el tamaño de grano es la temperatura; al respecto, Muchow (1990) mostró que las temperaturas altas aceleran el crecimiento del grano pero disminuyen el periodo de llenado del mismo y por tanto disminuyen también su peso individual. En ese sentido es claro que en los sitios PA, BA y TL las temperaturas altas (Figura 2B) durante el periodo de llenado de grano afectaron el tamaño de grano, mientras que en EZ las causas fueron las

Figura 2. Precipitación (A) y temperatura media máxima (B) en seis localidades de Estado de Tlaxcala, México. Año 2009, Enero (Ene)-Diciembre (Dic). HU = Huamantla; TL = Tlatempa; EZ = Emiliano Zapata; PA = Papalotla; NB = Nicolás Bravo; BA = Cuahuixmatlac. 
bajas temperaturas y la deficiencia de lluvia (Figura 2). En el sitio NB la deficiencia de agua después de la polinización inhibió sustancialmente la fotosíntesis y dio como resultado una escasa acumulación de materia seca, como postularon Boyer y Westgate (2004). Debido a sus granos de tamaño intermedio y pequeño, todos los híbridos son aptos para su procesamiento doméstico y por la IMT, ya que estos granos se hidratan con mayor facilidad que los grandes, lo que resulta en una mayor humedad de nixtamal, masa y tortilla, y por consiguiente un mayor rendimiento (Salinas et al., 2010).

En el peso hectolítrico, el modelo AMMI mostró significancia en el CP1 y CP2, componentes que explicaron 60.2 $\%$ de la SC de la interacción (31.7 y $28.4 \%$, respectivamente) (Cuadro 3). El híbrido más estable a través de todos los ambientes fue el 'H-72' [19]. Independientemente de la estabilidad, 11 híbridos mostraron significativamente mayor PH que la media general $\left(76.6 \mathrm{~kg} \mathrm{hL}^{-1}\right)$; los híbridos ' $\mathrm{H}-58^{\prime}$ [1], 'Niebla' [20] y 'H-48' [13] presentaron el PH más bajo (Figura 1C). Por su alto PH destacaron los genotipos 'Búho' [14] y 'H-55' [9], ambos con interacción negativa. Los híbridos más inestables a través de los ambientes fueron ' $\mathrm{H}$ 47' [6], 'AS-722' [17] y 'H-51' [8].

Las localidades de Nicolás Bravo, San Bartolomé, Tlatempa y Emiliano Zapata discriminaron bien entre genotipos. El mayor PH para la localidad de Nicolás Bravo se obtuvo en el híbrido 'AS-722” [17], para Tlatempa en el 'H-47' [6], para Emiliano Zapata en el 'H-51' [8] y para Cuahuixmatlac en el 'H-52' [12]. Según Yang et al. (2000), el tamaño del grano no influye en el peso hectolítrico, lo que aquí se comprobó con la baja correlación significativa entre estas variables $\left(r=0.55^{* *}\right)$. Estos mismos autores encontraron que en ambientes con alta precipitación el peso hectolítrico es bajo, y en los de baja precipitación el PH es relativamente alto, pero tal comportamiento no se observó en este experimento. Los ambientes donde se registró mayor tamaño de grano también fueron los de mayor rendimiento de grano y alto $\mathrm{PH}$, y viceversa. Todos los híbridos mostraron un $\mathrm{PH}$ mayor a $74 \mathrm{~kg} \mathrm{hL}^{-1}$, que es el mínimo demandado por la IMT y IHN.

En el IF los dos primeros CP fueron significativos. El CP1 explicó 49.4 \% de la SC de la IGA y el CP2 explicó $20.7 \%$ (Cuadro 3). De acuerdo con Medina et al. (2002), valores del CP1 con alrededor de $50 \%$ de la SC de la IGA es satisfactorio para el empleo de este componente como parámetro de estabilidad. Los híbridos más estables en cuanto a dureza del grano fueron 'H-49' [7] y 'H-58' [1]; los más inestables fueron 'Prospecto AE3' [18], 'H-47' [6] y 'H-70' [5] (Figura 1D). Todas las localidades discriminaron bien entre genotipos. Las localidades de Tlatempa y Huamantla presentaron granos de textura dura (IF $<37 \%$ ), mientras que en Papalotla los granos fueron de dureza intermedia (IF
$=38$ a $62 \%$ ), y en Emiliano Zapata, Cuahuixmatlac y Nicolás Bravo los granos fueron suaves (IF $>63 \%$ ).

De los 20 híbridos evaluados, tres ('H-64', 'H-55' y 'Búho') presentaron granos duros, 12 fueron de grano con dureza intermedia, y cinco de grano suave ('H-58' [1], 'H-48' [13], 'H-49' [7], 'Niebla' [20] y 'H-47' [6]). El IF correlacionó con: rendimiento $\left(\mathrm{r}=-0.60^{* *}\right), \mathrm{PH}\left(\mathrm{r}=-0.76^{\star *}\right)$, y PCG $(\mathrm{r}=$ $\left.-0.68^{\star *}\right)$, lo que significa que entre más duros sean los granos mayor será el rendimiento, el $\mathrm{PH}$ y el tamaño del grano. Ninguno de los materiales evaluados son aceptables para la IHN debido a que esta industria requiere granos muy duros y duros (IF $<20 \%$ ), mientras que para la IMT 15 de los 20 híbridos son aptos para la elaboración de tortillas, todos cultivados en las localidades de HU, TL y PA.

En cuanto al color del grano, el CP1 resultó significativo con $41.8 \%$ de la explicación de la IGA. De acuerdo con la Figura 1E, los híbridos más estables fueron 'H-58' [1], 'H-68' [4], 'Promesa' [15] y 'H-51' [8]. El 'H-48' [13] fue el híbrido más blanco ( $58 \%$ de reflectancia) y uno de los más estables. Solo ocho híbridos presentaron un color apropiado para la industria de harina nixtamalizada (' $\mathrm{H}-48$ ' [13], 'H-47' [6], 'H-58' [1], 'H-49' [7], 'H-51' [8], 'Niebla' [20], 'Prospecto AE3' [18] y 'Búho' [14]), con un porcentaje de reflectancia mayor a 55 \% (Vázquez et al., 2012). Entre localidades los granos más blancos correspondieron a los híbridos cultivados en NB, EZ y BA, y los más cremosos se produjeron en los sitios de PA, HU y TL. El color del grano se relacionó estrechamente con la dureza $\left(\mathrm{r}=0.68^{\star *}\right)$, los granos más blancos (\% de reflectancia más alto) fueron los más suaves (mayor contenido de endospermo harinoso) y los de menor \% de reflectancia fueron los más duros, comportamiento similar al observado por Salinas et al. (1992).

$\mathrm{El}$ análisis de varianza para el color de harina mostró diferencias significativas $(\mathrm{P}<0.01)$ por efecto de localidad, híbrido y por la interacción de ambos factores (Cuadro 3). Los valores más altos de color en harina se presentaron en las muestras de BA (84.7 \%) y los más bajos en PA. La industria de harina nixtamalizada prefiere granos que den una harina con reflectancia mayor a $77 \%$, y solo 11 híbridos presentaron un color de harina aceptable para este proceso ('H-64' [3], 'H-72' [19], 'H-70' [5], 'H-58' [1], 'Búho' [14], 'H-66' [2], 'AS-722' [17], 'H-50' [16], 'Niebla' [20], 'Prospecto AE3' [18], y 'H-40' [11]). Las harinas con reflectancia mayor a $77 \%$ se obtuvieron en los sitios $\mathrm{BA}, \mathrm{NB}$ y EZ, lo que coincide con su mayor suavidad y mayor valor de color en grano.

\section{Composición estructural y calidad nixtamalero-tortillera}

Se encontraron diferencias significativas $(\mathrm{P}<0.01)$ para 
el contenido de pedicelo, pericarpio y endospermo entre localidades, genotipos y por efecto de la interacción de ambos factores. El contenido de germen no mostró diferencias entre localidades (Cuadro 4). El contenido de pedicelo y pericarpio fue mayor en los híbridos cultivados en Bartolomé, mientras que el contenido de endospermo fue mayor en los cultivados en Huamantla. De acuerdo con Salinas et al. (2010), los híbridos de Huamantla satisfacen los requerimientos de estas estructuras para la IMT, los de Bartolomé tuvieron un contenido de pericarpio superior al $6 \%$ aceptado, pero en esta estructura se localizan las gomas naturales que imparten flexibilidad y elasticidad a las tortillas.

La humedad del nixtamal fue mayor en los híbridos de Bartolomé que en los de Huamantla (Cuadro 4), debido al menor tamaño de su grano y su mayor suavidad, lo cual permitió una mejor hidratación del endospermo del grano durante la nixtamalización y el reposo (Salinas et al., 2010). Los híbridos cultivados en Cuahuixmatlac retuvieron más pericarpio que los de Huamantla, lo que provocó una menor pérdida de sólidos, sin embargo, no rebasan el límite de $5 \%$ permitido por la industria (Vázquez et al., 2012). De acuerdo con Serna et al. (2008), ésta es una característica heredable y usualmente los granos más pequeños retienen mas pericarpio que los grandes, comportamiento similar al encontrado en este trabajo, ya que los granos de los híbridos cultivados en Bartolomé fueron más pequeños que los de Huamantla.

La humedad del nixtamal y masa de los híbridos de Bartolomé se reflejó en una mayor humedad de tortillas, con respecto a los de Huamantla, lo que resultó en un mayor rendimiento; sin embargo, no se alcanzó el rendimiento ideal para esta industria cuyo mínimo es de $1.5 \mathrm{~kg}$ de tortilla por kg de maíz. Según Salinas et al. (2010), para obtener un rendimiento de $1.5 \mathrm{~kg}$ de tortilla por $\mathrm{kg}$ de maíz se requiere al menos una humedad de $45 \%$ en tortillas. No se encontró correlación entre la textura de las tortillas (fuerza de ruptura) y su humedad, ya que de acuerdo con Salinas et al. (2010) las de mayor humedad son las de textura más suave. En cuanto al color de las tortillas recién hechas, con el colorímetro Agtron no se detectaron diferencias entre localidades, pero con el Hunter Lab pudo diferenciar tortillas un poco más blancas en Huamantla que en Cuahuixmatlac (Cuadro 5). Este mismo comportamiento se observó a las $24 \mathrm{~h}$, no obstante que en este tiempo las tortillas se tornaron ligeramente más oscuras.

\section{CONCLUSIONES}

El análisis AMMI proporcionó una buena descripción de la interacción genotipo $\mathrm{x}$ ambiente y de la estabilidad de los 20 híbridos, para todas las variables de calidad de grano. Las condiciones de siembra y las ambientales influyeron ampliamente sobre el rendimiento y las características físicas de los híbridos, especialmente sobre el tamaño del grano y la dureza. Los híbridos 'Prospecto AE3', 'H-55' y ' $\mathrm{H}-47$ ' fueron estables en rendimiento aunque éste fue bajo (5.3 a $5.5 \mathrm{t} \mathrm{ha}^{-1}$ ). Ningún híbrido cumplió las especificaciones de la industria de harinas nixtamalizada debido a la suavidad de su grano. Para la industria de la masa y tortilla 15 híbridos cultivados en Huamantla, Tlatempa y Papalotla

Cuadro 4. Composición estructural (en \%) de grano y calidad de nixtamal-masa para 20 híbridos de maíz evaluados en dos localidades del Estado de Tlaxcala, México. PV-2009.

\begin{tabular}{cccccccccc}
\hline \multirow{2}{*}{ Clave } & Pedicelo & Pericarpio & Germen & Endospermo & HN & PS & PR & HM \\
\cline { 2 - 9 } & \multicolumn{7}{c}{$\%$} & $\%$ & \\
\hline Huamantla & $1.4 \mathrm{~b}$ & $5.7 \mathrm{~b}$ & $10.0 \mathrm{a}$ & $82.8 \mathrm{a}$ & $44.1 \mathrm{~b}$ & $3.3 \mathrm{a}$ & $37.4 \mathrm{a}$ & $55.8 \mathrm{~b}$ \\
& & & & & & & & & \\
Bartolomé & $1.9 \mathrm{a}$ & $6.5 \mathrm{a}$ & $10.1 \mathrm{a}$ & $81.5 \mathrm{~b}$ & $46.3 \mathrm{a}$ & $3.2 \mathrm{~b}$ & $38.3 \mathrm{a}$ & $57.3 \mathrm{a}$ \\
\hline
\end{tabular}

Medias con letras iguales en cada columna no son estadísticamente diferentes (Tukey, 0.05). HN = humedad de nixtamal; PS = sólidos en nejayote; $\mathrm{PR}$ = pericarpio retenido; $\mathrm{HM}$ = humedad de masa.

Cuadro 5. Calidad de tortillas de 20 híbridos de maíz evaluados en Huamantla(HU) Cuahuixmatlac (BA), dos localidades del Estado de Tlaxcala, México. PV-2009.

\begin{tabular}{|c|c|c|c|c|c|c|c|c|c|}
\hline \multirow{2}{*}{ Localidad } & \multicolumn{5}{|c|}{ Tortillas recién hechas } & \multicolumn{4}{|c|}{ Tortillas $24 \mathrm{~h}$} \\
\hline & $\mathrm{H}(\%)$ & Rendimiento & Color (\% R) & Color (L) & FR (gf) & $\mathrm{H}(\%)$ & Color (\% R) & Color (L) & $\mathrm{FR}(\mathrm{gf})$ \\
\hline $\mathrm{HU}$ & $42.9 \mathrm{a}$ & $1.43 \mathrm{~b}$ & $82.6 \mathrm{a}$ & $75.3 \mathrm{a}$ & $156.2 \mathrm{a}$ & $42.7 \mathrm{a}$ & $81.6 \mathrm{a}$ & $74.7 \mathrm{a}$ & $469.4 \mathrm{a}$ \\
\hline $\mathrm{BA}$ & $44.1 \mathrm{a}$ & $1.47 \mathrm{a}$ & $82.6 \mathrm{a}$ & $75.0 \mathrm{~b}$ & $160.8 \mathrm{a}$ & $43.8 \mathrm{a}$ & $81.2 \mathrm{a}$ & $74.6 \mathrm{a}$ & $481.3 \mathrm{a}$ \\
\hline
\end{tabular}


cumplieron los requerimientos. Los ambientes de Nicolás Bravo, Emiliano Zapata y Cuahuixmatlac fueron los que afectaron más el rendimiento y la calidad del grano. Los híbridos con mayor estabilidad en rendimiento a través de localidades no fueron los de mejor calidad de grano para el proceso de nixtamalización.

\section{BIBLIOGRAFÍA}

AACC, American Association of Cereal Chemists (2000) Approved Methods of the AACC. 16th ed. AACC. The Association. St. Paul, MN. USA. $1200 \mathrm{p}$

Arámbula V G, J A Méndez A, J González H, E Gutiérrez A, E Moreno M (2004) Evaluación de una metodología para determinar características de textura de tortilla de maíz (Zea mays L.) Arch. Latinoam. Nutr. 54:216-222.

Arellano V J L, J Virgen V, M A Ávila P (2010) H-66 híbrido de maíz para los Valles Altos de los Estados de México y Tlaxcala. Rev. Mex. Cien. Agríc. 1:257-262.

Arellano V J L, J Virgen V, I Rojas M, M A Avila P (2011) H-70: Híbrido de maíz de alto rendimiento para temporal y riego del Altiplano Central de México. Rev. Mex. Cien. Agríc. 2:619-626.

Billeb S A C, R Bressani (2001) Características de cocción por nixtamalización de once variedades de maíz. Arch. Latinoam. Nutr. 51:86-94.

Boyer J S, M E Westgate (2004) Grain yields with limited water. J. Exp. Bot. 55:2385-2394

CONAGUA-SMN, Comisión Nacional del Agua (2012) Normales climatológicas por estación. Disponible en: http://smn.conagua.gob. $\mathrm{mx} /$ (Agosto 2012).

Córdova H (1992) Respuestas diferenciales para rendimiento de híbridos de maíz evaluados en ambientes contrastantes de Latinoamérica PCCMCA 1990. Agron. Mesoam. 3:1-8.

Hristov N, N Mladenov, V Djuric, A Kondic-Spika, A MarjanovicJeromela, D Simic (2010) Genotype by environment interactions in wheat quality breeding programs in southeast Europe. Euphytica 174:315-324.

INIFAP, Instituto Nacional de Investigaciones Forestales, Agrícolas y Pecuarias (2012) Red Nacional de Estaciones Estatales Agroclimatológicas. Disponible en: http://clima.inifap. gob.mx/redclima/ (Agosto 2012).

María R A, I Rojas M, M A Ávila P, J A Gámez V (2003) Producción de Maíz de Temporal en el Estado de Tlaxcala. Folleto para Productores Número 3. Instituto Nacional de Investigaciones Forestales Agrícolas y Pecuarias-Campo Experimental Tlaxcala. Tlaxcala. México. $16 \mathrm{p}$

Medina S, C Marín R, V Segovia, A Bejarano, Z Venero, R Ascanio, E Meléndez (2002) Evaluación de la estabilidad del rendimiento de variedades de maíz en siete localidades de Venezuela. Agron. Trop. 52:255-275.
Muchow R C (1990) Effect of high temperature on grain-growth in fieldgrown maize. Field Crops Res. 23:145-158.

Oikeh S O, A Menkir, B Maziya-Dixon, R M Welch, R P Glahn, G Gauch (2004) Environmental stability of iron and zinc concentrations in grain of elite early-maturing tropical maize genotypes grown under field conditions. J. Agric. Sci. 142:543-551.

Rharrabti Y, L F García M, D Villegas, C Royo (2003) Durum wheat quality in Mediterranean environments III. Stability and comparative methods in analyzing G x E interaction. Field Crops Res. 80:141-146.

Rincón T J A, S Castro N, J A López S, A J Huerta, C Trejo L, F Briones E (2006) Temperatura alta y estrés hídrico durante la floración en poblaciones de maíz tropical. Rev. Int. Bot. Exp. 75:31-40.

Salinas M Y, J L Arellano V, F Martínez B (1992) Propiedades físicas, químicas y correlaciones de maíces híbridos precoces para valles altos. Arch. Latinoam. Nutr. 42:161-167.

Salinas M Y, J J López R, G B González F, G Vázquez C (2007) Compuestos fenólicos del grano de maíz y su relación con el oscurecimiento de masa y tortilla. Agrociencia 41:295-305.

Salinas M Y, N O Gómez M, J E Cervantes M, M Sierra M, A Palafox C E Betanzos M, B Coutiño E (2010) Calidad nixtamalera y tortillera en maíces del trópico húmedo y sub-húmedo de México. Rev. Mex. Cien. Agríc. 1:509-523.

Salinas M Y, F J Cruz C, S A Díaz O, F Castillo G (2012) Granos de maíces pigmentados de Chiapas, características físicas, contenido de antocianinas y valor nutracéutico. Rev. Fitotec. Mex. 35:33-41.

Serna S S O, C A Amaya G, P Herrera M, J L Melesio C, R E Preciado O, A D Terron I, G Vázquez C (2008) Evaluation of the limecooking and tortilla making properties of quality protein maize hybrids grown in Mexico. Plant Foods Human Nutr. 63:119125 .

SIAP, Servicio de Información Agroalimentaria y Pesquera (2012) Cierre de la Producción Agrícola por Estado. SIAP-SAGARPA. Disponible en http://www.siap.gob.mx/ (Marzo 2012).

The C, H Calba, C Zonkeng, E L M Ngonkeu, V O Adetimirin, H A Mafouasson, S S Meka, W J Horst (2006) Responses of maize grain yield to changes in acid soil characteristics after soil amendments. Plant Soil 284:45-47.

Vázquez C G, S García L, Y Salinas M, D J Bergvinson, N Palacios R (2011) Grain and tortilla quality in landraces and improved maize grown in the highlands of Mexico. Plant Foods Human Nutr. 66:203-208.

Vázquez C M G, H Mejía A, C Tut C, N Gómez M (2012) Características de granos y tortillas de maíces de alta calidad proteínica desarrollados para los Valles Altos Centrales de México. Rev. Fitotec. Mex. 35:23-31.

Yang P, R J Shunk, A E Haken, Y X Niu, S H Zou, P Buriak, S R Eckhoff, E Tumbleson (2000) Yield, protein content, and viscosity of starch from wet-milled corn hybrids as influenced by environmentally induced changes in test weight. Cereal Chem. 77:44-47.

Zobel R W, M J Wright, H G Gauch J (1988) Statistical analysis of a yield trial. Agron. J. 80:388-393. 